\title{
High expression of immune checkpoints is associated with the TIL load, mutation rate and patient survival in colorectal cancer
}

\author{
MARA KITSOU $^{1}$, GEORGIOS D. AYIOMAMITIS ${ }^{2}$ and APOSTOLOS ZARAVINOS ${ }^{3}$ \\ ${ }^{1}$ Department of Life Sciences, School of Sciences, European University Cyprus, 1516 Nicosia, Cyprus; \\ ${ }^{2}$ First Department of Surgery, Tzaneio General Hospital, 18536 Piraeus, Greece; \\ ${ }^{3}$ College of Medicine, Member of QU Health, Qatar University, P.O. Box 2713 Doha, Qatar
}

Received February 10, 2020; Accepted April 13, 2020

DOI: $10.3892 /$ ijo.2020.5062

\begin{abstract}
Adoptive cell therapy with the use of tumorinfiltrating lymphocytes (TILs) is a very promising immunotherapeutic approach for the treatment of patients with colorectal cancer (CRC). However, within the tumor microenvironment, co-inhibitory immune checkpoints can inactivate TILs. The aim of the present study was to examine the association between the TIL load, the mutation rate and the clinical outcome in the immune landscape of patients with CRC. RNA-seq and whole exome seq data of 453 colon adenocarcinomas (COAD) and rectal adenocarcinomas (READ), along with the TIL load and clinicopathological information of each patient, were extracted from the TCGA GDC Data Portal and analyzed computationally. The expression of immune checkpoint molecules was compared between colon cancer and normal tissue. A total of 9 immune-related gene signatures were investigated in CRC. Spearman's correlation analysis was performed to examine the correlation between the TIL load with the expression of each immune checkpoint molecule. Indoleamine 2,3-dioxygenase 1 (IDO1) was found to be significantly overexpressed in CRC, whereas V-domain Ig suppressor of T cell activation (VISTA) and lymphocyte activating 3 (LAG3) were markedly downregulated. A high expression of cytotoxic T-lymphocyte-associated protein 4 (CTLA-4), IDOI, programmed cell death $1(P D-1)$ and T-cell immunoreceptor with Ig and ITIM domains (TIGIT), tended to be associated with a better overall survival of the patients. In COAD, the TIL load positively correlated with the expression of adenosine A2A receptor (ADORA2A), CTLA-4, hepatitis A virus cellular receptor 2 (HAVCR2), lymphocyte activating 3 (LAG3), programmed death-ligand $P D-L 1, P D-L 2, T I G I T$ and VISTA, whereas in READ, such positive correlations were noted only between the TIL load and $L A G 3$ or $P D-L 2$. The
\end{abstract}

Correspondence to: Dr Apostolos Zaravinos, College of Medicine, Member of QU Health, Qatar University, P.O. Box 2713 Doha, Qatar E-mail: azaravinos@qu.edu.qa

Key words: immune checkpoints, colorectal cancer, mutation rate, tumor-infiltrating lymphocyte load, patient survival 'central memory T-cell' and 'exhausted T-cell' gene signatures were significantly lower among the READ tumors. The expression of $P D-1, P D-L 1, P D-L 2, C T L A-4$ and $I D O 1$ was significantly higher among COAD patients with a high mutation rate ( $>34$ mutations/ $\mathrm{Mb}$ ) compared to those with a lower rate. Somatic mutations in $P D-1, P D-L 1, C T L A-4$ and other checkpoint molecules did not seem to affect their expression levels. On the whole, the data of the present study highlight the association of immune checkpoint molecules with the TIL load, patient survival and a high mutation rate in CRC. The data corroborate that patients with colon cancer with higher PDI, PD-L1/2, CTLA-4 and IDOI expression, and a high mutation rate, are the ones who will benefit more from the respective immune checkpoint inhibition therapies.

\section{Introduction}

Colorectal cancer (CRC) is the third most common type of cancer, with $\sim 1.4$ million cases diagnosed worldwide in 2012 (1). The prognosis of the disease largely depends on the stage of the tumor at diagnosis (2). The disease is relatively heterogenous, and is classified into 4 different consensus molecular subtypes. The main characteristic of CRC is genetic instability, which can be due to either chromosomal instability (CIN) (3) or microsatellite instability (MSI) due to a defective DNA mismatch repair (dMMR) system (4). Additionally, $\mathrm{CpG}$ island methylation phenotype (CIMP) is a feature that induces epigenetic instability by silencing through promoter hypermethylation of a range of tumor suppressor genes, including mutL homolog 1 (MLH1) (5).

Apart from the surgical removal of the tumor, usually followed by adjuvant 5-fluoruracil (5-FU)-based chemotherapy, various immunotherapeutic approaches are currently being investigated as alternative options for the treatment of the disease (6,7). Latest encouraging developments in cancer immunotherapy, which involve priming the host's natural immune defenses to recognize, target and destroy cancer cells effectively, have brought some glimpse of hope for combatting CRC (8-11). To this end, tremendous progress has been made in the understanding of the immune microenvironment of CRC (12). The deciphering of the immunological and molecular landscape of the tumor may help determine subsets of immunogenic CRC, and determine potential predictive markers to help 
select patients for immunotherapeutic approaches (11). Apart from malignant cells, a solid CRC tissue contains various other innate immune cells (granulocytes, mast cells and monocytes/macrophages), adaptive immune cells (T-cells and B-cells), fibroblasts and endothelial cells. Working together, these cells contribute to the inflammatory and/or immunological status of the tumor tissue via cell-to-cell contact and/or the production of cytokines and chemokines. Tumor-infiltrating lymphocytes (TILs) are mixtures of T-cells, B-cells, natural killer (NK) cells, macrophages and other innate cells in variable proportions, with T-cells being the most abundant (13). They can infiltrate the solid tumor, and are used as signals of the immune system, in its attempt to attack the cancer cells. Primarily, TILs appear in the human body to indicate the existence of the host, thus reflecting the dynamic process of cancer immunization $(14,15)$.

The present study aimed to investigate whether a high mutation rate is associated with distinct expression profiles of various immune checkpoint molecules. To this end, the association between the TIL load and overall survival of patients with CRC was first examined, determining the expression of various immune checkpoint molecules, including programmed death-ligands 1 and $2(P D-L 1 / 2)$, cytotoxic T-lymphocyte-associated protein 4 (CTLA-4) and indoleamine 2,3-dioxygenase 1 (IDO1). In addition, the TIL load and the expression of such immune checkpoint molecules in each tumor, including the tumor's mutation rate, were evaluated. Finally, 9 immune-related gene signatures were compared between CRC and normal tissue. The results provide evidence that high levels of PD1, PD-L1/L2, CTLA-4 and IDO-1 are associated with the TIL load, a high mutation rate and the overall survival of colon cancer patients.

\section{Materials and methods}

Data extraction and analysis. Next generation sequencing (NGS) and clinicopathological data for 453 colorectal adenocarcinoma patients were extracted from the Cancer Genome Atlas (TCGA-COAD and TCGA-READ datasets, containing colon and rectum adenocarcinomas, respectively) and the data were computationally examined.

The expression of a list of immune checkpoint molecules and other, prospective checkpoint molecules, including programmed cell death 1 (PD-1; PDCD1), PD-L1 (CD274), PD-L2 (PDCDILG2), CTLA-4, T-cell immunoreceptor with Ig and ITIM domains (TIGIT), IDO1, IDO2, lymphocyte activating 3 (LAG3), V-set domain-containing T-cell activation inhibitor 1 (VTCN1), V-domain Ig suppressor of $\mathrm{T}$ cell activation (VISTA), Ig-like transcript (ILT)2, ILT4 and human leukocyte antigen $\mathrm{G}(H L A-G)$ were analyzed using RNA-seq data of 275 COAD and 92 READ tumors, and these were compared to the gene expression data of a total of 349 normal colon and 318 normal rectum samples, which were extracted from the TCGA and GTEx projects. The expression levels of each gene were calculated in transcripts per million mapped reads (TPM), adding an offset of 0.1 , as previously described (16-18). One-way analysis of variance (ANOVA) was performed using the disease state (tumor or normal) as a variable to determine the statistically significant differentially expressed genes. The expression data were first log-transformed for differential analysis and the fold change $\left(\log _{2} \mathrm{FC}\right)$ was defined as the difference of the median value of the tumor samples from the median value of the normal samples. Genes with $\mid \log _{2} \mathrm{FC}>1 \mathrm{l}$ and $\mathrm{P}<0.01$ were considered as differentially expressed. The corresponding percentage (\%) of TIL ('percent_lymphocyte_infiltration') and tumor-associated neutrophilic (TAN) load ('percent_neutrophil_infiltration') of the patients with CRC were extracted from TCGA using the Genomic Data Commons (GDC) Data Portal (https://portal. gdc.cancer.gov/), as previously described (17) (Table SI).

Patient survival analysis. Survival analysis was based on the expression status of adenosine $\mathrm{A} 2 \mathrm{~A}$ receptor (ADORA2A), CD8, CTLA-4, hepatitis A virus cellular receptor 2 (HAVCR2), IDO1, IDO2, LAG3, PD1, PD-L1, PD-L2, TIGIT, VISTA and $V T C N 1$ or the multi-gene signatures. The overall patient survival was plotted on Kaplan-Meier curves using the Gene Expression Profiling Interactive Analysis (GEPIA2) web server (19). Differences in overall survival between high- and low gene-expressing patients were scored using the log-rank test. Spearman's correlation analysis was used to examine the correlation between the TIL load with the expression of each immune checkpoint molecule.

Immune-related gene signatures in $C R C$. The following immune-related gene signatures from GEPIA2 (19) were compared between the CRC tumor and normal samples, within each TCGA dataset: Naive T-cell [C-C motif chemokine receptor $(C C R) 7$, lymphoid enhancer-binding factor 1 ( $L E F 1)$, transcription factor 7 (TCF7) and L-selectin (SELL)]; effector T-cell [CX3C chemokine receptor $1(C X 3 C R 1)$, fibroblast growth factor binding protein 2 (FGFBP2) and Fc fragment of IgG receptor IIIa $(F C G R 3 A)]$; effector memory T-cell [PDCDI, dual specificity protein phosphatase 4 (DUSP4), granzyme $(G Z M) K$, GZMA and interferon gamma (IFNG)]; central memory T-cell [CCR7, SELL and interleukin $(I L) 7 R]$; resident memory T-cell [CD69, integrin, alpha E (ITGAE), C-X-C chemokine receptor type $6(C X C R 6)$ and myeloid-associated differentiation marker (MYADM)]; exhausted T-cell (HAVCR2, TIGIT, LAG3, PDCD1, $C X C L 13$ and $L A Y N$ ); resting Treg T-cell [forkhead box P3 (FOXP3), IL2RA); effector Treg T-cell [FOXP3, CTLA-4, $C C R 8$ and tumor necrosis factor (TFN) receptor superfamily member 9 (TNFRSF9)]; and Th1-like [CXCL13, HAVCR2, IFNG, CXCR3, basic helix-loop-helix family member e40 (BHLHE4O) and CD4]. For the analysis of gene signatures, the mean value of the $\log _{2}(\mathrm{TPM}+1)$ was used as the signature score and the CRC samples were compared against matched normal data from both the TCGA. The gene signatures with $\| \log _{2} \mathrm{FC}>11$ and $\mathrm{P}<0.01$ (ANOVA) were considered as significantly different between tumor and normal tissues.

Association between the mutation rate and the expression of immune checkpoint molecules in COAD. iCoMut Beta for FireBrowse was used to categorize COAD tumors into those having a low ( $<34$ mutations per $\mathrm{Mb}$ ) or high ( $>34$ mutations per $\mathrm{Mb}$ ) mutation rate. The expression of 5 widely-established immune checkpoint molecules (PD-1,PD-L1, PD-L2, CTLA-4 and $I D O 1$ ) was then compared between COAD with a 'high' and 'low' mutation rate. The data were analyzed using the $\mathrm{R}$ environment. 
Cell-type fractions within microsatellite stable (MSS) and instable (MSI) colon adenocarcinomas. The Cancer Immunome Database (TCIA) (20) was used to gain insight into the cell type fractions within MSS, MSI-low or MSI-high patients within the TCGA-COAD database. The MSI status was defined according to the Cancer Genome Atlas Network (21).

Detection of single nucleotide variants (SNVs) in immune checkpoint genes and association with their expression. The gene expression and mutations across the GDC-TCGA-COAD and READ datasets were explored through the UCSC Xena platform (22). Gene expression (RNAseq) was evaluated using the normalized HTSeq-Fragments Per Kilobase of transcript per million mapped reads (FPKM). Somatic SNVs and insertions/deletions (indels) (deleterious, splice, missense/inframe, silent and complex or unannotated mutations) across immune checkpoint genes were called using MuTect2 (v.4.1) variant aggregation and masking. The GRCh37 build of the human reference genome was used for analysis.

\section{Results}

Elevated levels of CTLA-4, HAVCR2, IDO1, PD-1, PD-L1, $P D-L 2, T I G I T, V T C N 1$ and HLA-G were detected in COAD and READ tumors against their corresponding normal tissues. However, the difference did not reach statistical significance. Among these checkpoint molecules, IDOI was exceptionally upregulated both in COAD and READ. On the other hand, ILT2, ADORA2A, LAG3 and VISTA exhibited a lower expression in CRC compared to normal tissue. In the case of $L A G 3$ and VISTA, the difference reached statistical significance $(\mathrm{P}<0.01)$ (Fig. 1).

Patients with CRC expressing high levels of CD $8 A$, CTLA-4, IDOI, PD-1 and TIGIT, exhibited a better overall survival compared to patients expressing with low levels of these molecules (Fig. 2). This overexpression correlated with the TIL load in both datasets. Specifically, among the COAD tumors, the TIL load positively correlated with the expression of CD8, as well as that of ADORA2A, CTLA-4, HAVCR2, LAG3, $P D-L 1, P D-L 2, T I G I T$ and VISTA $(\mathrm{P}<0.005$, Spearman's correlation analysis) (Fig. 3A). On the other hand, among the READ tumors, such positive correlations between the TIL load and the expression of immune checkpoint molecules (or the TCR co-receptor marker $C D 8 A$, which acts on the recognition of antigens displayed by an antigen presenting cell in the context of MHC-I molecules), were scored only for $L A G 3$ and $P D-L 2$ (Fig. 3B). Of note, the expression of CTLA-4 significantly correlated with that of the remaining immune checkpoint molecules in COAD, indicating that immune response in colon tumors elicits multiple host and tumor mechanisms of immune suppression in the tumor microenvironment, other than the PD1/PD-L1 axis. Therefore, this observation supports the hypothesis that a combinatorial targeting of multiple immune checkpoint pathways may expand the clinical benefit for these patients (17) (Fig. 3C).

Although the outcome of patients with CRC has improved significantly with the recent implementation of annual screening programs, reliable prognostic biomarkers are still required due to the heterogeneity of the disease. Cumulative evidence indicates an association between immune signature and prognosis of the disease. Therefore, the present study explored 9 immune-related gene signatures in CRC and compared them to normal tissue from the TCGA and GTEx projects. A significantly lower expression of the 'central memory T-cell' and 'exhausted T-cell'- related gene signatures, was found in READ tumors. The 'resting and effector Treg T-cell', 'nä̈ve T-cell' and 'Thl-like' gene signatures were enriched among both CRC subtypes (COAD and READ), although without reaching statistical significance. On the other hand, the 'resident memory T-cell' signature revealed lower levels in the CRC samples compared to the normal tissue. Overall, these findings reveal significant differences in the immune-related gene signatures between colorectal tumors and normal tissue, reflecting their association with the prognosis of the disease (Fig. 4A).

The second subtype of CRC (CMS2) contains hypermutated, microsatellite instable (MSI+) tumors, with a strong immune activation. MSI occurs due to a defective DNA mismatch repair (dMMR), which accumulates a high number of mutations (23). A higher mutational load (and hence a higher neoepitope load) is positively associated with overall TIL infiltration, memory T cells, and CRC-specific survival (24). Herein, differences were found in the percentage (\%) of different cell types between tumors, based on their MSI status. Specifically, MSI-high tumors contained a higher percentage of $\mathrm{M} 1$ macrophages $(35 \%)$ and $\mathrm{CD}^{+} \mathrm{T}$ cells $(7 \%)$ compared to MSS (26\% M1 macrophages and 3\% CD8 ${ }^{+} \mathrm{T}$ cells) or MSI-low CRCs (25\% M1 macrophages and 3\% CD8 ${ }^{+} \mathrm{T}$ cells). On the other hand, MSI-high CRCs contained less neutrophils (25\%) compared to MSS (33\%) or MSI-low (32\%) tumors (Fig. 4B).

In addition, the $\mathrm{CRC}$ tumors were stratified based on their mutation rate and the association of the expression of immune checkpoint molecules with the corresponding TIL load (\%) was investigated in each tumor. Tumors with a high mutational rate (>34 mutations/Mb) exhibited the same mutational signature profile, i.e., a preference for $* \mathrm{CpG}>\mathrm{T}$ mutations, with those having a low mutational rate ( $<34$ mutations/Mb) (Fig. 5A). Overall, 25 genes were recurrently mutated in CRC, exhibiting elevated mutation rates among hypermutated tumors. The significantly mutated genes in the hypermutated cancers included APC, TP53, PIK3CA, PTEN, KRAS, ATM, SYNE1, SMAD4, FBXW7, KIT, BRAF, PTCH1, CSMD3, NF1, RB1, among others at a lower frequency ( $<100$ mutations) (Fig. 5A). A significantly higher $P D-1, P D-L 1, P D-L 2, C T L A-4$ and $I D O 1$ expression was found among the hypermutated colon adenocarcinomas, compared to those with a lower mutation rate (Fig. 5B). These data suggest that a high (synonymous and non-synonymous) tumor mutation rate seems to be associated with clinical benefit in patients who receive anti-PD1, anti-PD-L1 or anti-CTLA-4 therapy. Of major interest, it was found that the TIL load (\%) was also significantly higher $(\mathrm{P}=0.021)$ among the hypermutated tumors, suggesting that part of these mutations, belonging to cancer neoepitopes, might be recognized by TILs that are in immediate contact with the tumor cells (Fig. 5B).

In addition, the existence of somatic mutations in $P D-1$ (PDCD1), PD-L1 (CD274), CTLA-4 and other checkpoint genes was investigated. A total of $100 \mathrm{SNVs}$ and Indels were detected, containing missense/inframe, deleterious, silent, or intron/RNA somatic point mutations within the 12 immune checkpoint 


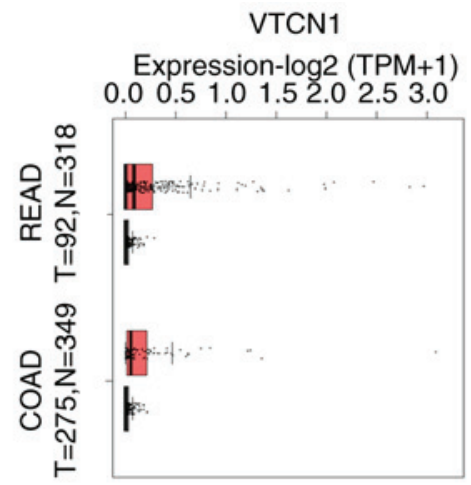

ILT2

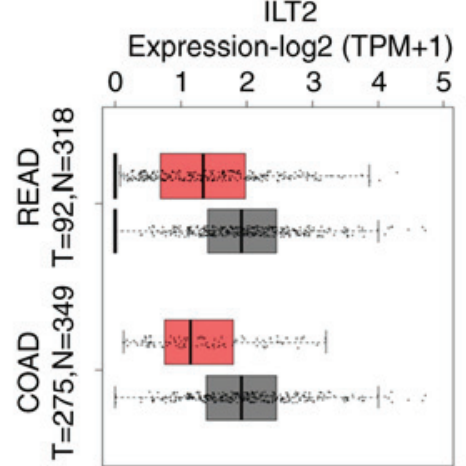

ILT4

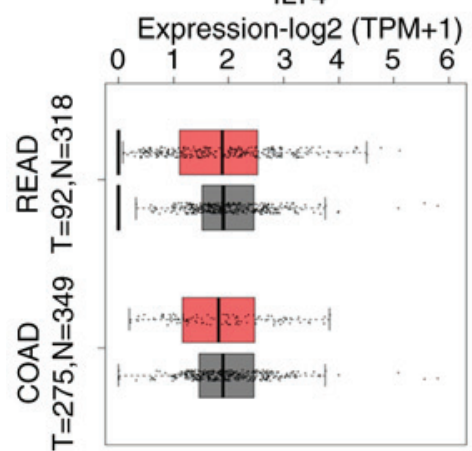

HLA-G

Expression-log2 (TPM+1)

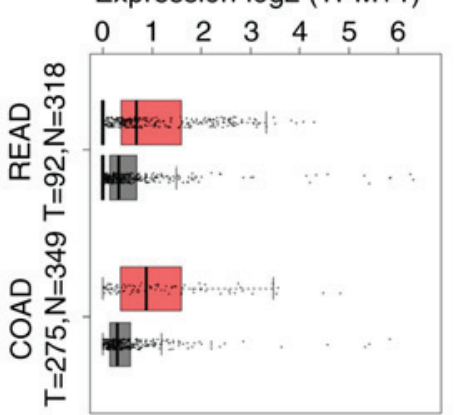

CD274 (PD-L1)
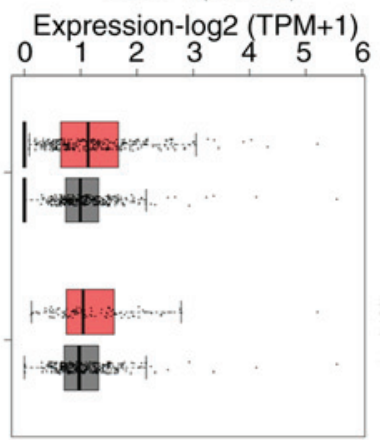

PDCDL1G2 (PD-L2)

Expression-log2 (TPM+1)
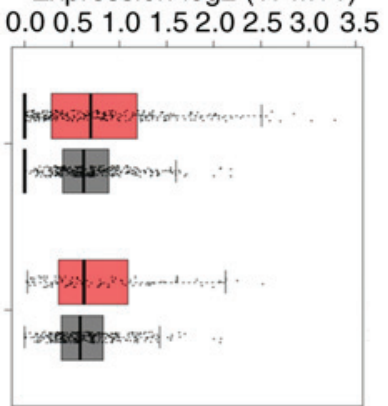

TIGIT
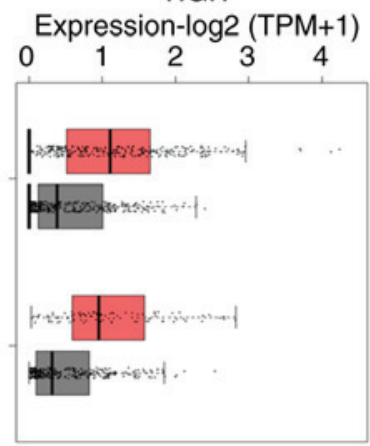

C10orf54 (VISTA)
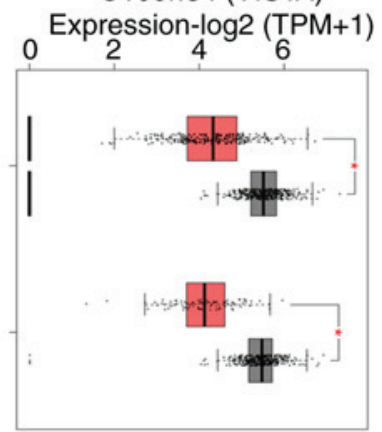

IDO1

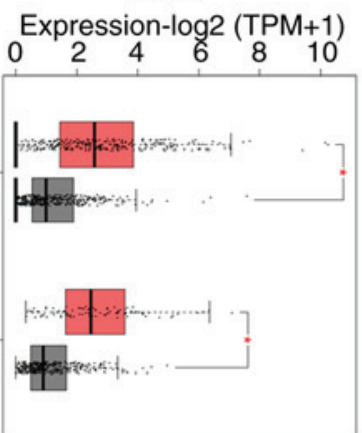

IDO2
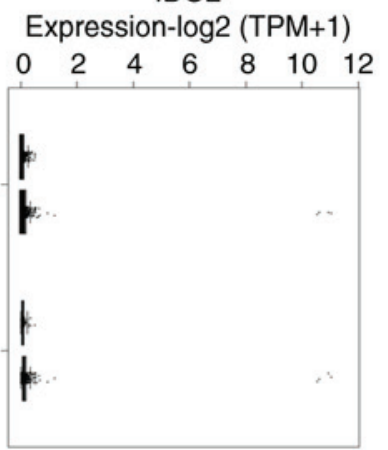

LAG3

Expression- $\log 2(\mathrm{TPM}+1)$
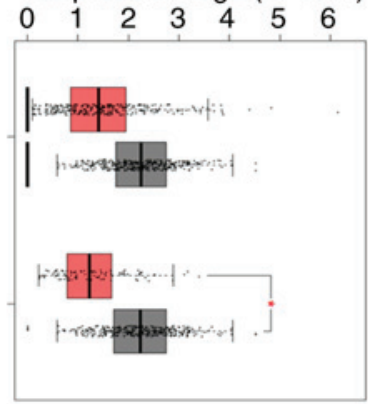

PDCD1 (PD-1)
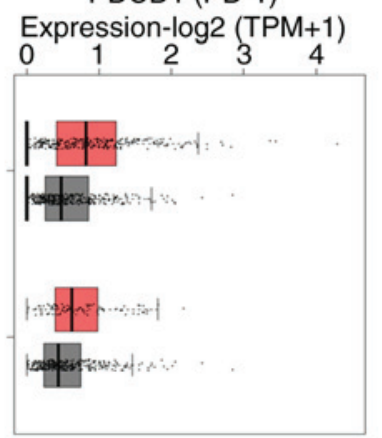

ADORA2A

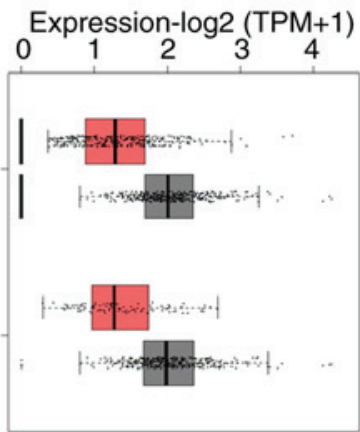

CD8

Expression-log2 (TPM+1)

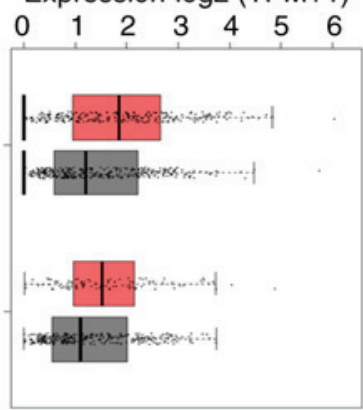

CTLA-4

Expression-log2 (TPM+1)

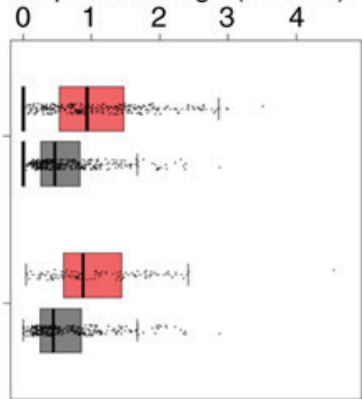

HAVCE2

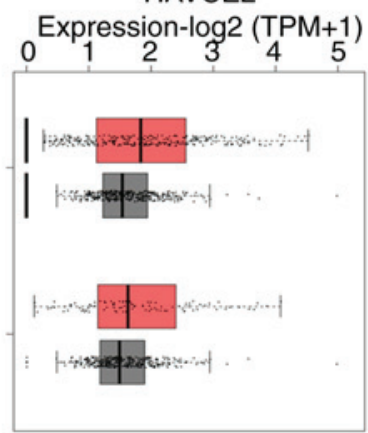

Figure 1. Among the immune checkpoints analyzed, IDOI was significantly upregulated in CRC, whereas, LAG3 and VISTA were significantly downregulated. The higher levels of CTLA-4, HAVCR2, IDO1, PD-1, PD-L1, PD-L2, TIGIT, VTCN1 and HLA-G in CRC did not reach statistical significance. Equally, the lower levels of ILT2 and ADORA2A in CRC did not reach statistical significance. Red stars denote statistically significant differences $(\mathrm{P}<0.01)$ between COAD (or READ tumors) and the normal tissue from the TCGA and GTEx projects. CRC, colorectal cancer; COAD, colon adenocarcinomas; READ, rectal adenocarcinomas.

molecules of interest, across the COAD and READ tumors (Table SII). All variants were randomly distributed and did not seem to associate with the corresponding expression levels of each gene (Figs. 6 and S1). Therefore, these results indicate that gene expression is not driven by mutations in these checkpoint genes.

\section{Discussion}

Immunoediting has turned out to be critical in appreciating the immune system's ability to harness tumor growth and spread in several types of cancer $(25,26)$. New immune-based 

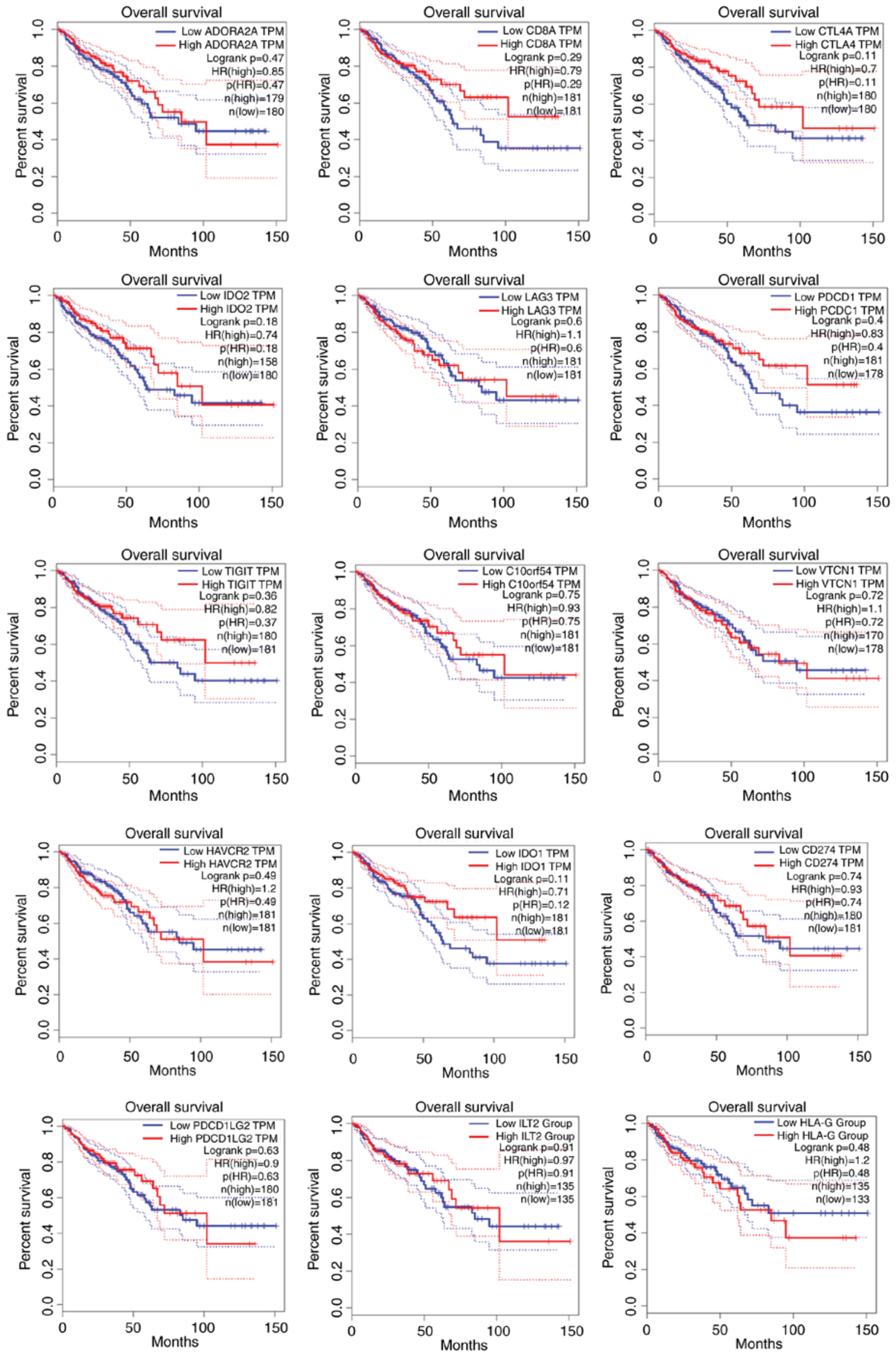

Figure 2. Overall survival curves of patients with CRC, expressing high or low levels of the immune checkpoint molecules ADORA2A, CTLA-4, HAVCR2, IDO1/2, LAG3, PD-1 (PDCD1), PD-L1 (CD274), PD-L2 (PDCD1LG2), TIGIT, VISTA (C10orf54), HLA-G, ILT2 and VTCN1, or the TCR co-receptor marker $C D 8$. The patients' high $C D 8, C T L A-4, I D O 1, P D 1$ and TIGIT expression levels, exhibited a tendency for improved overall survival, compared to those with low levels of the corresponding genes (log rank, $\mathrm{P}>0.05$ ). CRC, colorectal cancer. 

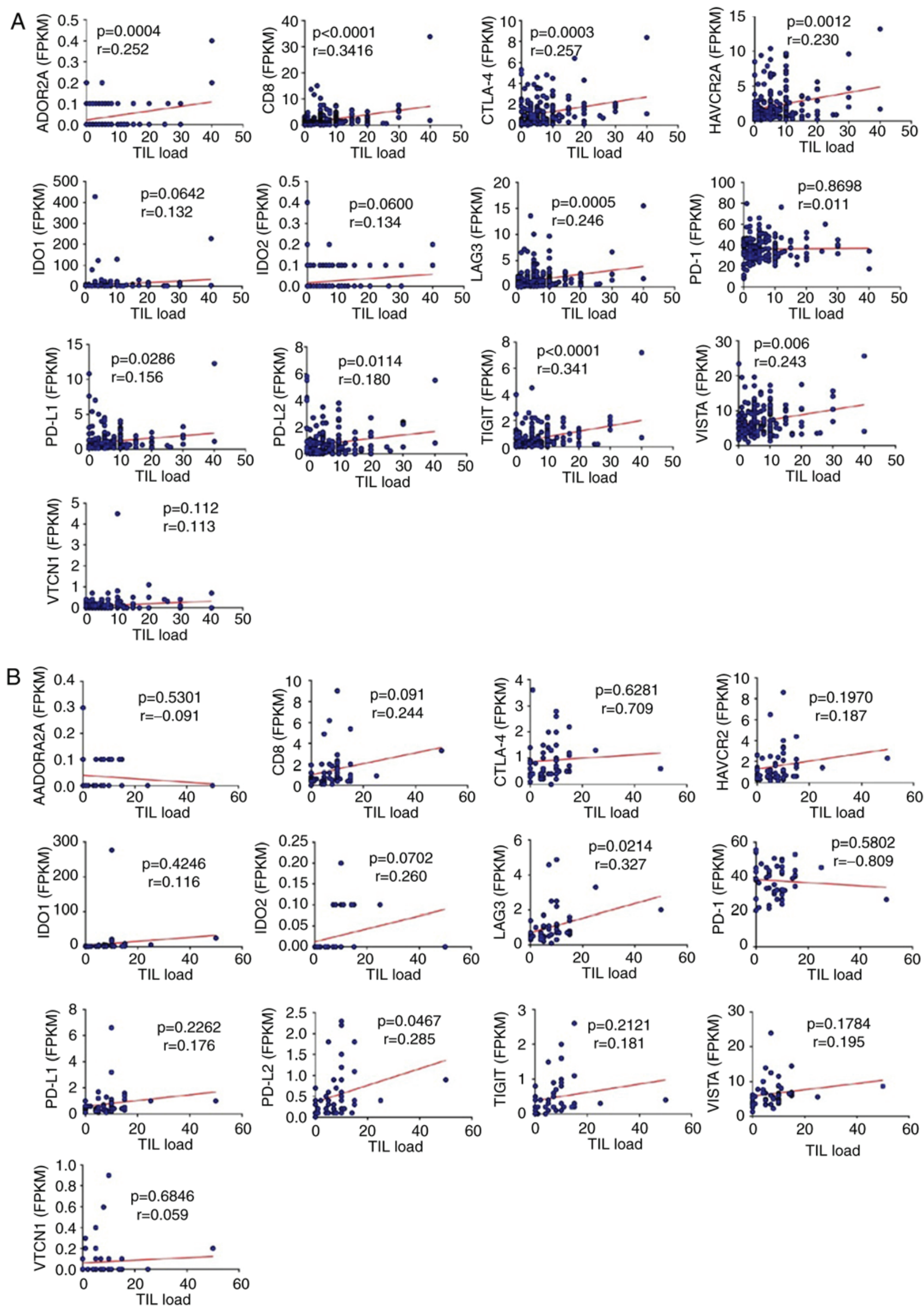

Figure 3. Correlation between the expression of immune checkpoints and the patients' corresponding TIL load, in (A) COAD and (B) READ tumors, respectively. 

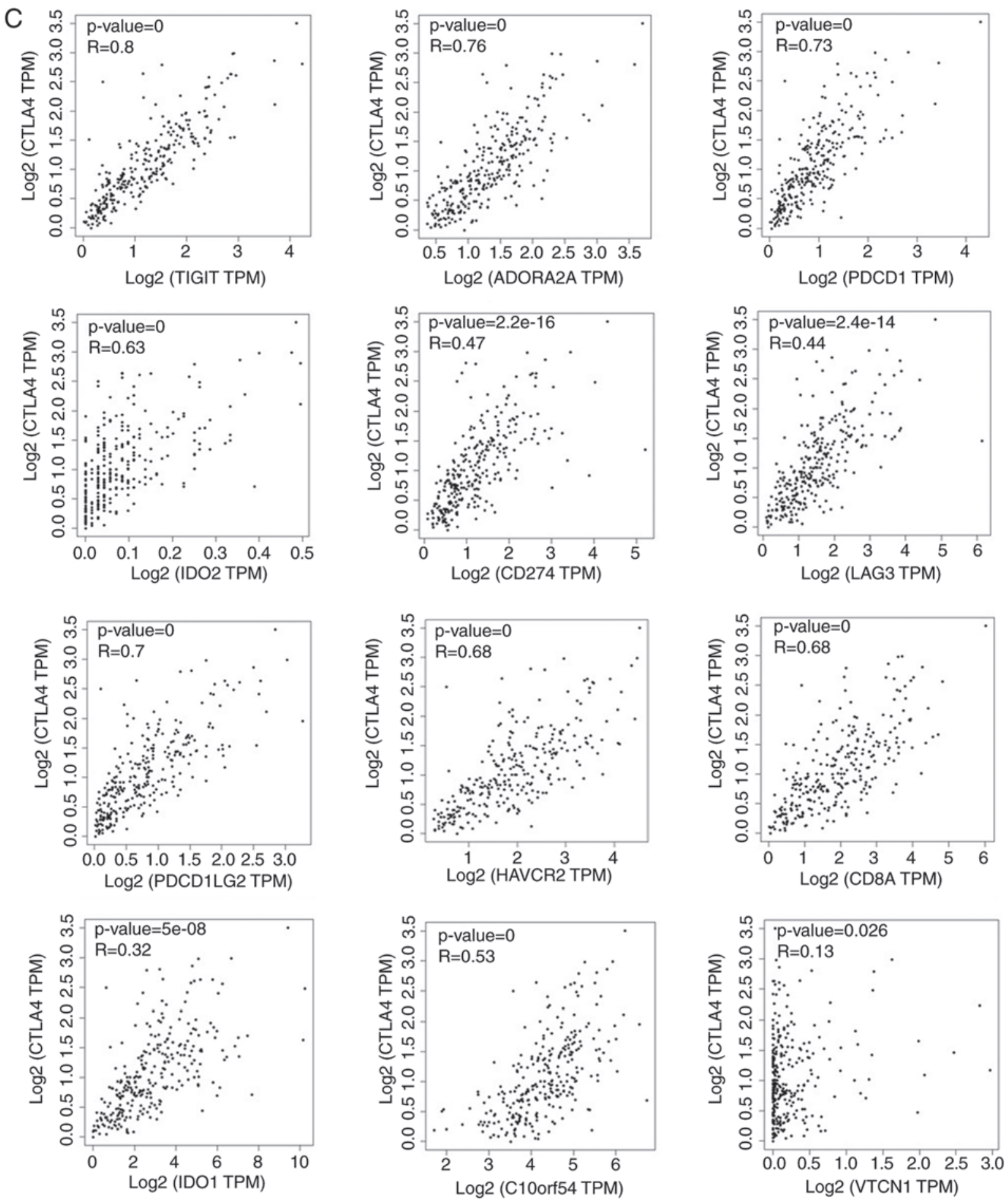

Figure 3. Continued. (C) Correlation between the expression of $C T L A-4$ and other immune checkpoint molecules, or the TCR co-receptor marker $C D 8$, in COAD. Spearman's correlation analysis was used with a P-value cut-off of 0.05 . COAD, colon adenocarcinomas; READ, rectal adenocarcinomas.

therapies have been recently proposed as treatment against primary and metastatic CRC, using either PD-1, PD-L1 and CTLA-4 inhibitors, or a combination of them in refractory (MSI-H and MSS) colorectal tumors $(13,27)$. In addition, adoptive cell therapy, using TILs from patients or donors, or differentiated from stem cells, is a highly promising immunotherapeutic strategy for CRC patients (28). These immune cells are then activated and expanded in vitro, and subjected to gene modification, before finally being infused back into the patients (28). Recently, Baek and Kim (29) obtained TILs from patients with CRC and evaluated their potential as an immunotherapeutic modality. They demonstrated that the ex vivo expanded TILs contained mostly effector memory T-cells and they were found to elicit an anti-tumor response. However, within the tumor microenvironment, the expression of co-inhibitory immune checkpoints can lead to the inactivation of such TILs (30).

In the present study, the expression of several immune checkpoints between CRC and normal tissue was compared, using data extracted from the TCGA and GTEx platforms. 

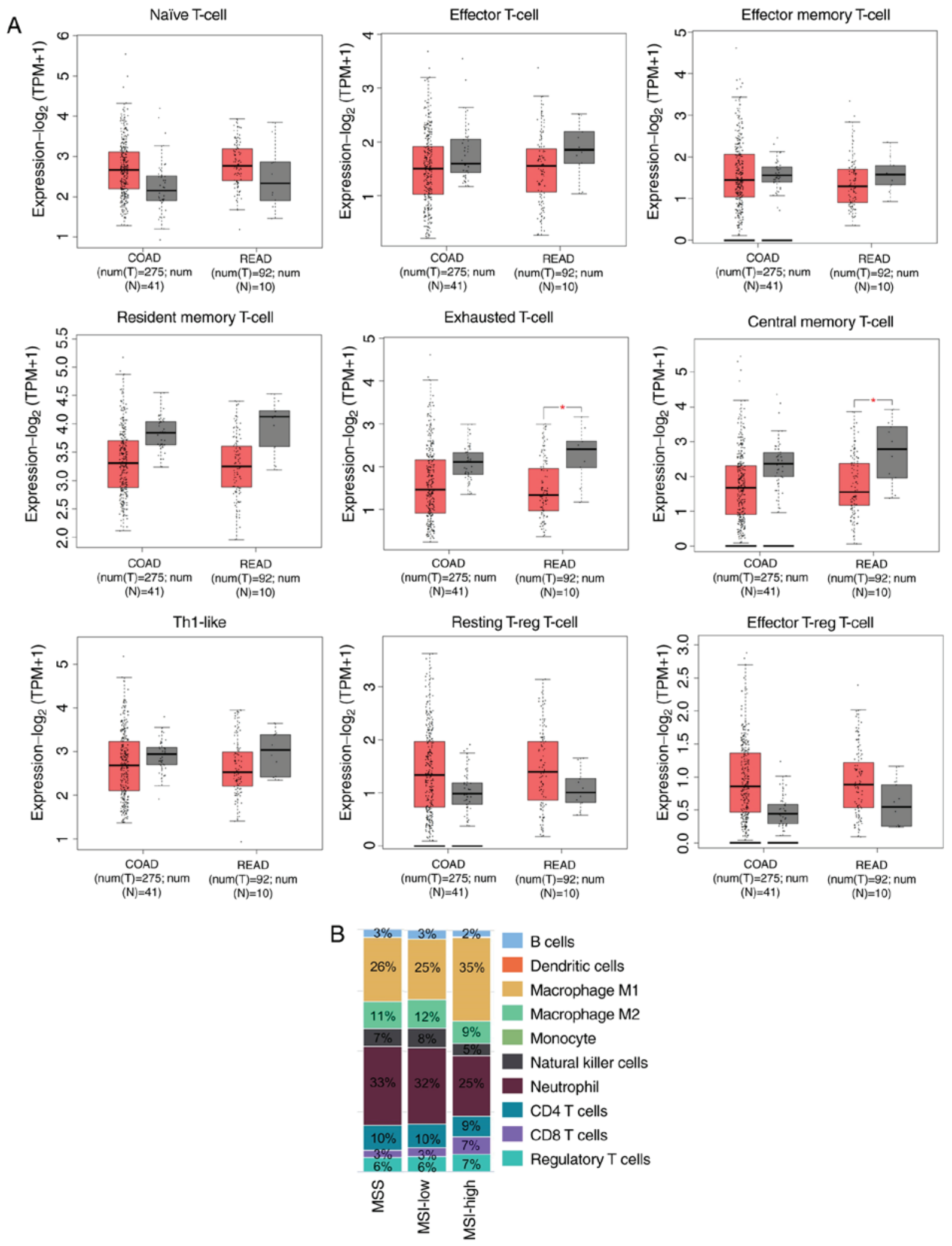

Figure 4. (A) Immune-related gene signatures between COAD (or READ) and normal tissue retrieved from the TCGA. The genes pertaining to each immune gene signature are denoted in the Materials and Methods. (B) Cell-type fractions were compared between MSS, MSI-low and MSI-high CRC patients through the Cancer Immunome Database (TCIA). MSI-high CRCs contained higher percentage of M1 macrophages (35\%) compared to MSS and MSI-low tumors (26 and 25\%, respectively). MSI-high CRCs also contained less neutrophils (25\%) compared to MSS and MSI-low tumors ( 33 and $32 \%$, respectively). COAD, colon adenocarcinomas; READ, rectal adenocarcinomas.

In addition, their association with patient survival, TIL load and the mutation rate of each tumor and was evaluated. Furthermore, the expression of different immune-related gene signatures in CRC compared to the normal tissue was investigated. A higher percentage of the so-called 'tumor preventing' M1 macrophages and CD8 ${ }^{+} \mathrm{T}$-cells was found 
Mutation rate
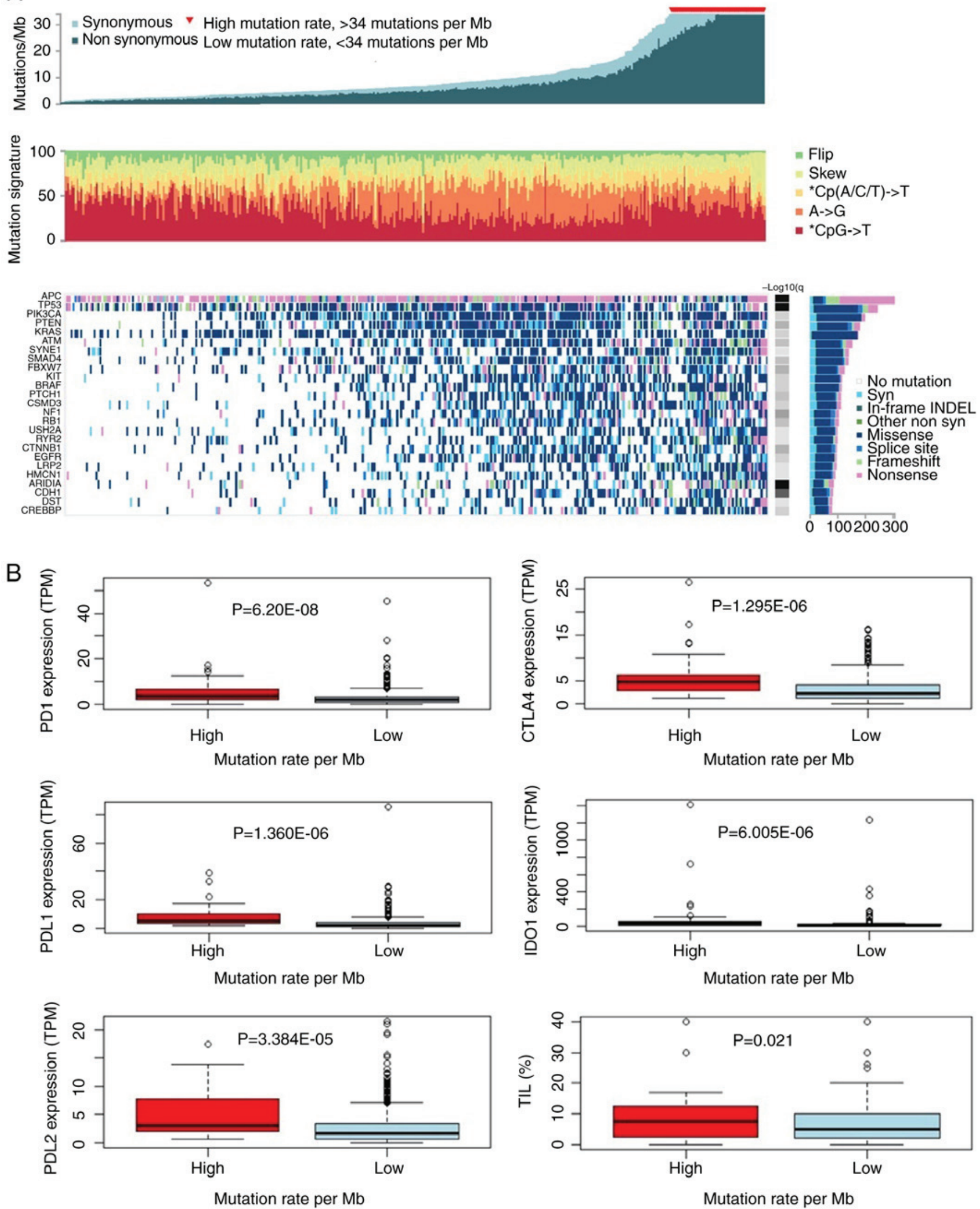

Figure 5. (A) COAD tumors were stratified to those having a high ( $>34$ mutations/Mb) or a low ( $<34$ mutations/Mb) mutation rate. Mutational signatures did not differ between hyper-mutated and non-hypermutated CRC tumors, both having a preference for $* \mathrm{CpG}>\mathrm{T}$ mutations. The long tail graph shows the 25 significantly mutated genes in hyper-mutated and non-hypermutated tumors. The significantly mutated genes among hypermutated tumors included $A P C, T P 53$, PIK3CA, PTEN, KRAS, ATM, SYNE1, SMAD4, FBXW7, KIT, BRAF, PTCH1, CSMD3, NF1, RB1, among others. Both mutational signatures and significantly mutated genes were assessed using iCoMut Beta for FireBrowse. (B) The expression levels of $P D-1, P D-L 1, P D-L 2, C T L A-4$ and $I D O 1$ were significantly higher among colon adenocarcinomas with a high mutation rate per Mb. The TIL (\%) load was significantly higher among tumors with a high mutation rate $(\mathrm{P}=0.021)$. COAD, colon adenocarcinomas; READ, rectal adenocarcinomas. 

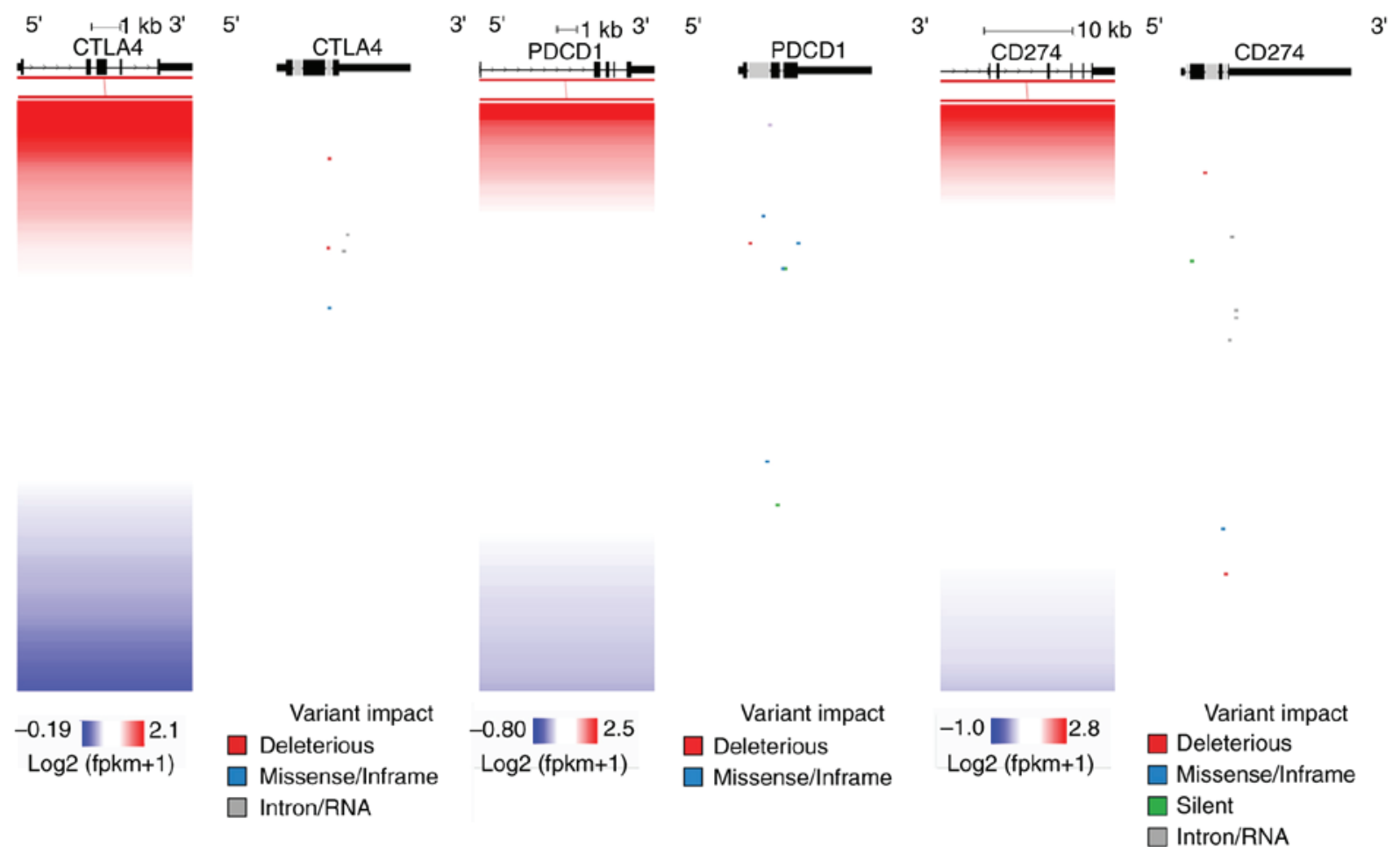

Figure 6. Gene expression levels of $C T L A-4, P D C D 1(P D-1)$ and $C D 274$ (PD-L1) do not seem to associate with the somatic mutation (SNPs and small INDELs). Gene expression values were measured in $\log _{2}(\mathrm{FPKM}+1)$ values and somatic mutations were analyzed using MuTect2 variant aggregation and masking.

among MSI-high tumors, compared to the MSI-low or MSS ones. In addition, the percentage of the 'tumor promoting' M2 macrophages, as well as that of neutrophils was lower in MSI-high tumors compared to the other two microsatellite groups, in accordance with reports that have previously associated these with an improved survival of patients with MSI-high CRC (31-33). A lower percentage of neutrophils was also found among MSI-high tumors. This is in agreement with a previous report by the authors demonstrating that patients with CRC with a low TAN percentage have an improved survival compared to those with a higher TAN load (17).

By stratifying patients with colon cancer based on their mutation rate (mutations per $\mathrm{Mb}$ ), it was found that those having a high mutation rate expressed significantly higher levels of $P D-1, P D-L 1 / L 2, I D O 1$ and $C T L A-4$. These observations are in agreement with those of previous reports (34-39), indicating that these patients may benefit more from a corresponding immune checkpoint blockade therapy. Therefore, the quantification of the mutational burden in these patients may be used as a predictive biomarker of immunotherapy via checkpoint inhibition. The expression of PD-L1 and TMB was recently found to have non-overlapping effects on the response rate to PD-1/PD-L1 inhibitors and was proposed that it can be used to categorize the immunologic subtypes of different tumor types, including CRC (37). In addition, the authors of the present study previously demonstrated that the protein levels of PD-1, PD-L1, PD-L2 and CTLA-4, similar to the CD8 marker, were significantly higher in dMMR/MSI-H CRCs, compared to dMMR/MSI-L and pMMR-MSS tumors. These observations indicate the influence that these immune checkpoint-expressing cells have on the tumor microenvironment by regulating immune responses (17).

The data of the present study revealed an enrichment of IDOI in CRC, highlighting its prominent role in the tumor microenvironment. Along with IDOI, CRC tumors expressed high levels of further immune checkpoint molecules, including CTLA-4 and PD-1. On the other hand, a low expression of ADORA2A, LAG3 and VISTA was found in CRC. This may be in contrast to the recent study by Xie et al (40), who found that VISTA protein was highly expressed in CRC; but this was mainly due to TILs. Therefore, it seems that VISTA (C10orf54) is indeed, downregulated in CRC, compared to $C T L A-4$ and $P D-1$. In accordance with the data of the present study, Lee et al (41) found a low percentage (23.6\%) of CRCs expressing LAG3. The blockade of LAG3 was also found to enhance tumor-infiltrating T-cell responses of mismatch repair-proficient (pMMR) liver metastasis of CRC, and was suggested as a new promising immunotherapeutic target for these tumors (42).

An increased mutational load in CRC was previously associated with other metrics, including high cytolytic activity, the count of MHC-I cancer neoepitopes, high microsatellite instability and deregulated expression of several immune checkpoints (17). The tumor's mutational burden was recently suggested to be predictive of the patients' response to immune checkpoint inhibition in MSI-high metastatic CRC (43). Herein, higher levels of $P D-1, P D-L 1$, $P D-L 2, C T L A-4$ and IDOI were also found among hypermutated colorectal tumors, indicating an association with the clinical benefit in patients who receive anti-PD1, anti-PD-L1 
or anti-CTLA-4 therapy. Importantly, it was found that the TIL load was significantly higher among tumors with a high mutation rate. Overall, the lymphocytic score was previously associated with the better survival of patients with CRC (44). In a similar study, Giannakis et al found an association between a higher neoantigen load and increased overall lymphocytic score in CRC (24). These observations date back even earlier, when Jass et al demonstrated that a high TIL load was an independent factor for the survival of patients with rectal cancer (45), and later on, Ogino et al demonstrated that higher levels of lymphocytic reactions and TILs were associated with patient prognosis (44). All these observations confirm that the presence of a high level of lymphoid reaction in the CRC tissue is associated with an improved prognosis.

The molecular landscape of CRC was previously characterized by the Cancer Genome Atlas Network (21), predicting the significantly mutated genes in CRC. In addition to their role in affecting normal cell function, tumor somatic mutations can generate neoantigens, which can be recognized by the host immune system (46). It was found that a high mutation rate was significantly associated with a high TIL load in these CRC tumors. This result is consistent with previous reports, and shows that patients with a big number of immunogenic mutations have an increased survival (47). Moreover, the corresponding tumors had higher cytotoxic T-cell (CTL) content, inferred from the expression of $C D 8 A$.

Overall, the findings of the present study highlight the association of immune checkpoints with the TIL load, patient survival and high mutation rate in CRC. The data corroborate that patients with colon cancer with a higher $P D 1, P D-L 1 / 2$, $C T L A-4$ and $I D O-1$ expression, and a high mutation rate, are the ones who will benefit more from the respective immune checkpoint inhibition therapies.

\section{Acknowledgements}

The authors would like to acknowledge the platforms TCGA, HPA, Broad Institute GDAC and The Cancer Immunome Atlas for downloading genetic and clinical data of the CRC patients.

\section{Funding}

No funding was received.

\section{Availability of data and materials}

All data generated or analyzed during this study are included in this published article [and its supplementary information files].

\section{Authors' contributions}

MK acquired and analyzed the data. GDA was involved in the conception and design of the study and critically reviewed the manuscript. AZ developed the methodology, and analyzed and interpreted the data; AZ also wrote the manuscript and supervised the study. All authors read and approved the final manuscript.

\section{Ethics approval and consent to participate}

Not applicable.

\section{Patient consent for publication}

Not applicable.

\section{Competing interests}

The authors declare that they have no competing interests.

\section{References}

1. Ferlay J, Soerjomataram I, Dikshit R, Eser S, Mathers C, Rebelo M, Parkin DM, Forman D and Bray F: Cancer incidence and mortality worldwide: Sources, methods and Major patterns in GLOBOCAN 2012. Int J Cancer 136: E359-E386, 2015.

2. Siegel RL, Miller KD, Fedewa SA, Ahnen DJ, Meester RGS, Barzi A and Jemal A: Colorectal cancer statistics, 2017. CA Cancer J Clin 67: 177-193, 2017.

3. Poulogiannis G, Ichimura K, Hamoudi RA, Luo F, Leung SY, Yuen ST, Harrison DJ, Wyllie AH and Arends MJ: Prognostic relevance of DNA copy number changes in colorectal cancer. J Pathol 220: 338-347, 2010

4. Arends MJ: Pathways of colorectal carcinogenesis. Appl Immunohistochem Mol Morphol 21: 97-102, 2013.

5. Ibrahim AE, Arends MJ, Silva AL, Wyllie AH, Greger L, Ito Y, Vowler SL, Huang TH, Tavaré S, Murrell A and Brenton JD: Sequential DNA methylation changes are associated with DNMT3B overexpression in colorectal neoplastic progression. Gut 60: 499-508, 2011.

6. Falzone L, Salomone S and Libra M: Evolution of cancer pharmacological treatments at the turn of the third millennium. Front Pharmacol 9: 1300, 2018.

7. Ganesh K, Stadler ZK, Cercek A, Mendelsohn RB, Shia J, Segal NH and Diaz LA Jr: Immunotherapy in colorectal cancer: Rationale, challenges and potential. Nat Rev Gastroenterol Hepatol 16: 361-375, 2019.

8. Kalyan A, Kircher S, Shah H, Mulcahy M and Benson A: Updates on immunotherapy for colorectal cancer. J Gastrointest Oncol 9: 160-169, 2018.

9. Copur MS: Immunotherapy in colorectal cancer. Oncology (Williston Park) 33: 686506, 2019.

10. Abakushina EV, Gelm YV, Pasova IA and Bazhin AV: Immunotherapeutic approaches for the treatment of colorectal cancer. Biochemistry (Mosc) 84: 720-728, 2019.

11. Dawood S: The evolving role of immune oncology in colorectal cancer. Chin Clin Oncol 7: 17, 2018.

12. Christofi T, Baritaki S, Falzone L, Libra M and Zaravinos A: Current perspectives in cancer immunotherapy. Cancers (Basel) 11: E1472, 2019

13. Koi M and Carethers JM: The colorectal cancer immune microenvironment and approach to immunotherapies. Futur Oncol 13: 1633-1647, 2017.

14. Chen DS and Mellman I: Elements of cancer immunity and the cancer-immune set point. Nature 541: 321-330, 2017.

15. Chen DS and Mellman I: Oncology meets immunology: The cancer-immunity cycle. Immunity 39: 1-10, 2013.

16. Ayiomamitis GD, Notas G, Vasilakaki T, Tsavari A, Vederaki S, Theodosopoulos T, Kouroumalis E and Zaravinos A: Understanding the interplay between COX-2 and hTERT in colorectal cancer using a multi-omics analysis. Cancers (Basel) 11: E1536, 2019.

17. Zaravinos A, Roufas C, Nagara M, de Lucas Moreno B Oblovatskaya M, Efstathiades C, Dimopoulos C and Ayiomamitis GD: Cytolytic activity correlates with the mutational burden and deregulated expression of immune checkpoints in colorectal cancer. J Exp Clin Cancer Res 38: 364, 2019.

18. Roufas C, Chasiotis D, Makris A, Efstathiades C, Dimopoulos C and Zaravinos A: The expression and prognostic impact of immune cytolytic activity-related markers in human malignancies: A comprehensive meta-analysis. Front Oncol 8: 27, 2018.

19. Tang Z, Kang B, Li C, Chen T and Zhang Z: GEPIA2: An enhanced web server for large-scale expression profiling and interactive analysis. Nucleic Acids Res 47: W556-W560, 2019. 
20. Charoentong P, Finotello F, Angelova M, Mayer C, Efremova M, Rieder D, Hackl H and Trajanoski Z: Pan-cancer immunogenomic analyses reveal genotype-immunophenotype relationships and predictors of response to checkpoint blockade. Cell Rep 18: 248-262, 2017

21. Cancer Genome Atlas Network: Comprehensive molecular characterization of human colon and rectal cancer. Nature 487: 330-337, 2012.

22. Goldman M, Craft B, Hastie M, Repečka K, Kamath A, McDade F, Rogers D, Brooks AN, Zhu J and Haussler D: The UCSC Xena platform for public and private cancer genomics data visualization and interpretation. bioRxiv 326470, 2019.

23. Guinney J, Dienstmann R, Wang X, de Reyniès A, Schlicker A, Soneson C, Marisa L, Roepman P, Nyamundanda G, Angelino P, et al: The consensus molecular subtypes of colorectal cancer. Nat Med 21: 1350-1356, 2015.

24. Giannakis M, Mu XJ, Shukla SA, Qian ZR, Cohen O, Nishihara R, Bahl S, Cao Y, Amin-Mansour A, Yamauchi M, et al: Genomic correlates of immune-cell infiltrates in colorectal carcinoma. Cell Rep 15: 857-865, 2016.

25. Swann JB and Smyth MJ: Immune surveillance of tumors. J Clin Invest 117: 1137-1146, 2007.

26. Ribatti D: The concept of immune surveillance against tumors. The first theories. Oncotarget 8: 7175-7180, 2017.

27. Boland PM and Ma WW: Immunotherapy for colorectal cancer. Cancers (Basel) 9: E50, 2017.

28. Fan J, Shang D, Han B, Song J, Chen H and Yang JM: Adoptive cell transfer: Is it a promising immunotherapy for colorectal cancer? Theranostics 8: 5784-5800, 2018.

29. Baek JH and Kim KJ: Expansion of tumor-infiltrating lymphocytes and their potential for application to adoptive cell therapy in patients with colorectal cancer. Eur J Surg Oncol 45: e58-e59, 2019.

30. Kodumudi KN, Siegel J, Weber AM, Scott E, Sarnaik AA and Pilon-Thomas S: Immune checkpoint blockade to improve tumor infiltrating lymphocytes for adoptive cell therapy. PLoS One 11: e0153053, 2016.

31. Edin S, Wikberg ML, Dahlin AM, Rutegård J, Öberg Å, Oldenborg PA and Palmqvist R: The distribution of macrophages with a M1 or M2 phenotype in relation to prognosis and the molecular characteristics of colorectal cancer. PLoS One 7 : e47045, 2012.

32. Narayanan S, Kawaguchi T, Peng X, Qi Q, Liu S, Yan L and Takabe K: Tumor infiltrating lymphocytes and macrophages improve survival in microsatellite unstable colorectal cancer. Sci Rep 9: 13455, 2019.

33. Zhao Y, Ge X, Xu X, Yu S, Wang J and Sun L: Prognostic value and clinicopathological roles of phenotypes of tumour-associated macrophages in colorectal cancer. J Cancer Res Clin Oncol 145 : 3005-3019, 2019

34. Rosenbaum MW, Bledsoe JR, Morales-Oyarvide V, Huynh TG and Mino-Kenudson M: PD-L1 expression in colorectal cancer is associated with microsatellite instability, BRAF mutation, medullary morphology and cytotoxic tumor-infiltrating lymphocytes. Mod Pathol 29: 1104-1112, 2016.

35. Fabrizio DA, George TJ Jr, Dunne RF, Frampton G, Sun J, Gowen K, Kennedy M, Greenbowe J, Schrock AB, Hezel AF, et al: Beyond microsatellite testing: Assessment of tumor mutational burden identifies subsets of colorectal cancer who may respond to immune checkpoint inhibition. J Gastrointest Oncol 9: 610-617, 2018.
36. Chan TA, Yarchoan M, Jaffee E, Swanton C, Quezada SA, Stenzinger A and Peters S: Development of tumor mutation burden as an immunotherapy biomarker: Utility for the oncology clinic. Ann Oncol 30: 44-56, 2019.

37. Yarchoan M, Albacker LA, Hopkins AC, Montesion M, Murugesan K, Vithayathil TT, Zaidi N, Azad NS, Laheru DA, Frampton GM and Jaffee EM: PD-L1 expression and tumor mutational burden are independent biomarkers in most cancers. JCI Insight 4: 126908, 2019.

38. Lal N, Beggs AD, Willcox BE and Middleton GW: An immunogenomic stratification of colorectal cancer: Implications for development of targeted immunotherapy. Oncoimmunology 4: e976052, 2015.

39. Hamada T, Soong TR, Masugi Y, Kosumi K, Nowak JA, da Silva A, Mu XJ, Twombly TS, Koh H, Yang J, et al: TIME (Tumor Immunity in the MicroEnvironment) classification based on tumor CD274 (PD-L1) expression status and tumor-infiltrating lymphocytes in colorectal carcinomas. Oncoimmunology 7: e1442999, 2018.

40. Xie S, Huang J, Qiao Q, Zang W, Hong S, Tan H, Dong C, Yang Z and Ni L: Expression of the inhibitory B7 family molecule VISTA in human colorectal carcinoma tumors. Cancer Immunol Immunother 67: 1685-1694, 2018.

41. Lee SJ, Byeon SJ, Lee J, Park SH, Park JO, Park YS, Kang WK, Lim HY, Kim KM and Kim ST: LAG3 in solid tumors as a potential novel immunotherapy target. J Immunother 42: 279-283, 2019.

42. Zhou G, Noordam L, Sprengers D, Doukas M, Boor PPC, van Beek AA, Erkens R, Mancham S, Grünhagen D, Menon AG, et al: Blockade of LAG3 enhances responses of tumor-infiltrating $\mathrm{T}$ cells in mismatch repair-proficient liver metastases of colorectal cancer. Oncoimmunology 7: e1448332, 2018.

43. Schrock AB, Ouyang C, Sandhu J, Sokol E, Jin D, Ross JS, Miller VA, Lim D, Amanam I, Chao J, et al: Tumor mutational burden is predictive of response to immune checkpoint inhibitors in MSI-high metastatic colorectal cancer. Ann Oncol 30: 1096-1103, 2019.

44. Ogino S, Nosho K, Irahara N, Meyerhardt JA, Baba Y, Shima K, Glickman JN, Ferrone CR, Mino-Kenudson M, Tanaka N, et al: Lymphocytic reaction to colorectal cancer is associated with longer survival, independent of lymph node count, microsatellite instability, and $\mathrm{CpG}$ island methylator phenotype. Clin Cancer Res 15: 6412-6420, 2009

45. Jass JR: Lymphocytic infiltration and survival in rectal cancer J Clin Pathol 39: 585-589, 1986.

46. Schumacher TN and Schreiber RD: Neoantigens in cancer immunotherapy. Science 348: 69-74, 2015.

47. Brown SD, Warren RL, Gibb EA, Martin SD, Spinelli JJ, Nelson BH and Holt RA: Neo-antigens predicted by tumor genome meta-analysis correlate with increased patient survival. Genome Res 24: 743-750, 2014.

This work is licensed under a Creative Commons Attribution-NonCommercial-NoDerivatives 4.0 International (CC BY-NC-ND 4.0) License. 